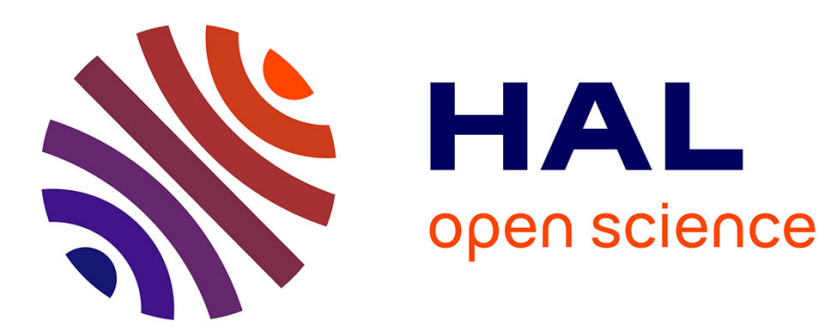

\title{
The structural sensitivity of open shear flows calculated with a local stability analysis
}

\author{
Matthew Juniper, Benoît Pier
}

\section{To cite this version:}

Matthew Juniper, Benoit Pier. The structural sensitivity of open shear flows calculated with a local stability analysis. European Journal of Mechanics - B/Fluids, 2015, 49, pp.426-437. 10.1016/j.euromechflu.2014.05.011. hal-01084708

\section{HAL Id: hal-01084708 \\ https://hal.science/hal-01084708}

Submitted on 5 Dec 2014

HAL is a multi-disciplinary open access archive for the deposit and dissemination of scientific research documents, whether they are published or not. The documents may come from teaching and research institutions in France or abroad, or from public or private research centers.
L'archive ouverte pluridisciplinaire HAL, est destinée au dépôt et à la diffusion de documents scientifiques de niveau recherche, publiés ou non, émanant des établissements d'enseignement et de recherche français ou étrangers, des laboratoires publics ou privés. 


\title{
The structural sensitivity of open shear flows calculated with a local stability analysis
}

\author{
Matthew P. Juniper ${ }^{\mathrm{a}, *}$, Benoit Pier ${ }^{\mathrm{b}}$ \\ ${ }^{a}$ Department of Engineering, University of Cambridge, Trumpington Street, Cambridge, CB2 1PZ, UK \\ ${ }^{\mathrm{b}}$ Laboratoire de mécanique des fluides et d'acoustique, École centrale de Lyon - CNRS - Université Claude-Bernard Lyon 1 - INSA Lyon, 36 avenue \\ Guy-de-Collongue, 69134, Écully, France
}

\section{A R T I C L E I N F O}

\section{Article history:}

Available online 5 June 2014

\section{Keywords:}

Stability

Structural sensitivity

Adjoint

Global modes

\begin{abstract}
A B S T R A C T
The structural sensitivity shows where an instability of a fluid flow is most sensitive to changes in internal feedback mechanisms. It is formed from the overlap of the flow's direct and adjoint global modes. These global modes are usually calculated with 2D or 3D global stability analyses, which can be very computationally expensive. For weakly non-parallel flows the direct global mode can also be calculated with a local stability analysis, which is orders of magnitude cheaper. In this theoretical paper we show that, if the direct global mode has been calculated with a local analysis, then the adjoint global mode follows at little extra cost. We also show that the maximum of the structural sensitivity is the location at which the local $k^{+}$and $k^{-}$branches have the same imaginary value. Finally, we use the local analysis to derive the structural sensitivity of two flows: a confined co-flow wake at $R e=400$, for which it works very well, and the flow behind a cylinder at $R e=50$, for which it works reasonably well. As expected, we find that the local analysis becomes less accurate when the flow becomes less parallel.
\end{abstract}

(c) 2014 Elsevier Masson SAS. All rights reserved.

\section{Introduction}

Many open flows have a steady solution to the Navier-Stokes equations that becomes unstable above a critical Reynolds number. Usually this instability is driven by one region of the flow, which is called the wavemaker region. The rest of the flow merely responds to forcing from this region. The shape, linear growth rate, and frequency of the instability can be calculated by considering the evolution of small perturbations about the steady solution. This is known as the direct global mode. The direct global mode emanates from the wavemaker region and grows spatially downstream, reaching a maximum at the streamwise location where the spatial growth rate is zero. For example, in the case of the flow behind a cylinder, this direct global mode is a sinuous flapping motion, whose nonlinear development is the familiar Kármán vortex street [1].

The receptivity of the direct global mode to harmonic open loop forcing is given by the last term in Eq. (9) of Ref. [2] and Eq. (7) of Ref. [3]. This term is proportional to the adjoint global mode, which is calculated in the same way as the direct global mode,

\footnotetext{
* Corresponding author. Tel.: +44 1223332585.

E-mail address: mpj1001@cam.ac.uk (M.P. Juniper).
}

but from the adjoint (rather than direct) linearized Navier-Stokes equations. If the perturbation magnitude is measured by the perturbation kinetic energy, which is the conventional approach, then there are only two significant differences between the direct and adjoint equations $[2,4]$. The first is the sign of the convection term, $V_{j} \partial v_{i} / \partial x_{j}$, and is called convective non-normality. The second is the appearance of a transconjugate operator, $v_{j} \partial V_{j} / \partial x_{i}$, and is called component-type non-normality, For the flows in this paper, the non-normality is almost entirely convective [4]. In a manner analogous to the direct global mode, the adjoint global mode emanates from the wavemaker region but grows spatially upstream, reaching a maximum at the streamwise location where the adjoint spatial growth rate is zero, or when it meets the upstream boundary. Physically, this reflects the fact that an open loop forcing signal will have most influence on the flow if it impinges on the wavemaker region, and if it is amplified by the flow before it does so.

The sensitivity of the direct global mode to changes in the linearized Navier-Stokes (LNS) equations is given by the penultimate term in Eq. (9) of Ref. [2]. This term is proportional to the overlap between the direct and adjoint global modes and is known as the structural sensitivity. It is equivalent to the sensitivity of the direct global mode to closed-loop feedback between the perturbation and the governing equations in the special case where the sensor and actuator are co-located. For example, in the case of the 
flow behind a cylinder, it can quantify the sensitivity of the flow to the presence of a small control cylinder that produces a small force on the flow in the opposite direction to the velocity perturbation $[5,6]$. Given that the direct global mode grows downstream of the wavemaker region and that the adjoint global mode grows upstream, the structural sensitivity is clearly maximal in the wavemaker region itself. Indeed, the wavemaker region is often defined as the position of maximum structural sensitivity, although alternative definitions exist [4, Section 4.2.1]. Physically, this reflects the fact that, for a closed loop feedback mechanism to be effective, it requires firstly that the perturbation has significant amplitude at that point, which is quantified by the direct global mode, and secondly that the flow has significant receptivity at that point, which is quantified by the adjoint global mode.

The above concepts were first introduced for the flow behind a cylinder at $R e=50$ by Hill [5] and Giannetti and Luchini [6,7] and have been extended to include the sensitivity to steady forcing and modifications to the base flow [8-10]. They have also been applied to recirculation bubbles [11] bluff bodies, both incompressible [12] and compressible [13], backward-facing steps [14], forward-facing steps [15], confined wakes [16,17], and a recirculation bubble in a swirling flow [18].

The direct global mode is usually found with a global stability analysis. This typically proceeds in three steps: (i) the NavierStokes (N-S) equations are linearized around a steady laminar flow, which is called the base flow and which is usually unstable; (ii) the equations are discretized and expressed as a 2D or 3D matrix eigenvalue problem; (iii) the most unstable eigenmodes are calculated with an iterative technique, such as an Arnoldi algorithm or power iteration. Each eigenmode consists of a complex eigenvalue, which describes the frequency and growth rate, and an eigenfunction, which describes the 2D or 3D shape that grows on top of the base flow until nonlinear effects become significant. As more elaborate configurations are examined, the number of degrees of freedom rapidly approaches millions, so global stability analyses can be extremely computationally expensive [4].

If the base flow varies slowly in the streamwise direction then the global stability analysis can be replaced with a local stability analysis [19]. The WKBJ approximation reduces the LNS equations over the entire domain into a series of local LNS or Orr-Sommerfeld $(\mathrm{O}-\mathrm{S})$ equations at each streamwise location. Each local equation can be discretized and expressed as a small matrix eigenvalue problem, which represents the dispersion relation between the complex frequency, $\omega$, and the complex wavenumber, $k$. At each streamwise location, the value of $\omega$ is found for which the dispersion relation is satisfied and for which $\mathrm{d} \omega / \mathrm{d} k=0$. This is known as the absolute complex frequency, $\omega_{0}$ and its imaginary part, $\omega_{0 i}$, is the absolute growth rate. The flow is absolutely unstable in regions in which $\omega_{0 i}$ is positive. These regions exist in every flow that is globally unstable due to hydrodynamic feedback. The frequency and growth rate of the linear global mode can be derived from the streamwise distribution of $\omega_{0}$. This also gives a specific spatial position for the region of the flow that, in the context of the local analysis, is known as the wavemaker [20]. Local stability analyses are much quicker and require much less computer memory than global stability analyses because they convert one large matrix eigenvalue problem into several small independent matrix eigenvalue problems. This is why they have been used so widely in the past and why they are still used for flows that are beyond the range of global analyses [21-23].

In all existing papers, the adjoint global mode is calculated with a global stability analysis. The purpose of this paper is to show that, if a local stability analysis is used to calculate the direct global mode, then the adjoint global mode follows at almost no extra cost. This means that, for weakly nonparallel flows, adjoint global modes and structural sensitivities can be estimated quickly and cheaply, without deriving the adjoint equations. After defining the form of the direct and adjoint equations in Section 2, we derive this result rigorously in Section 3 for the Ginzburg-Landau equation (G-L), which is often used as a simple model for slowly-developing flows. We then apply this to the linearized $\mathrm{N}-\mathrm{S}$ equations in Section 4 and demonstrate this on two flows in Section 5: a slowly-developing confined wake, and the flow behind a cylinder at $R e=50$.

\section{General form of the direct and adjoint equations}

Many different conventions are used to describe direct and adjoint global modes. The convention used here is similar to that used for local stability analysis, so that it is easy to compare the local and global approaches. It differs from that used in Hill $[5,24]$ and Giannetti and Luchini [6] in three ways. The direct and adjoint governing equations (1) and (2) have the same form so that their $k^{+}$and $k^{-}$branches in the local analysis have the same physical meaning. The adjoint variables are denoted with $\dagger$, rather than + or $*$, so that they are not confused with the $k^{+}$branch or with the complex conjugate. The inner product contains a complex conjugate so that the inner product of a complex state variable with itself is a real number.

The linearized governing equations are expressed in terms of the direct state variable, $\psi(x, t)$, the adjoint state variable, $\psi^{\dagger}(x, t)$, the direct linear spatial operator $L$, and the adjoint linear spatial operator $\mathrm{L}^{\dagger}$ :

$$
\begin{aligned}
& \frac{\partial \psi}{\partial t}-\mathrm{L} \psi=0, \\
& \frac{\partial \psi^{\dagger}}{\partial t}-\mathrm{L}^{\dagger} \psi^{\dagger}=0 .
\end{aligned}
$$

(The relationship between the direct and adjoint quantities will be specified in (8), after the inner product (7) has been defined.) Solutions to the initial value problems defined by (1) and (2) can be expressed for $t \in[0, \infty)$ as the sum of the direct and adjoint global modes:

$$
\begin{aligned}
& \psi(x, t)=\sum_{m} \hat{\psi}_{m}(x) \exp \left(-\mathrm{i} \omega_{m} t\right), \\
& \psi^{\dagger}(x, t)=\sum_{n} \hat{\psi}_{n}^{\dagger}(x) \exp \left(-\mathrm{i} \omega_{n} t\right) .
\end{aligned}
$$

Substituting (3) into (1) and (4) into (2) gives, for each mode,

$-\mathrm{i} \omega_{m} \hat{\psi}_{m}-\mathrm{L} \hat{\psi}_{m}=0$,

$-\mathrm{i} \omega_{n} \hat{\psi}_{n}^{\dagger}-\mathrm{L}^{\dagger} \hat{\psi}_{n}^{\dagger}=0$.

An inner product between state variables $f$ and $g$ is defined as

$\langle f, g\rangle \equiv \int_{-\infty}^{+\infty} f^{*} g \mathrm{~d} x$.

If boundary terms are assumed to be zero, as in Giannetti and Luchini [6], Hill [5], then the relationship between the direct operator, $\mathrm{L}$, and its adjoint, $\mathrm{L}^{\dagger}$, is given by

$\left\langle\mathrm{L} \hat{\psi}_{m}, \hat{\psi}_{n}^{\dagger}\right\rangle=\left\langle\hat{\psi}_{m}, \mathrm{~L}^{\dagger} \hat{\psi}_{n}^{\dagger}\right\rangle$.

These definitions determine the relationship between $\omega_{m}$ and $\omega_{n}$ :

$\left\langle\mathrm{L} \hat{\psi}_{m}, \hat{\psi}_{n}^{\dagger}\right\rangle=\left\langle\hat{\psi}_{m}, \mathrm{~L}^{\dagger} \hat{\psi}_{n}^{\dagger}\right\rangle$,

$\left\langle-\mathrm{i} \omega_{m} \hat{\psi}_{m}, \hat{\psi}_{n}^{\dagger}\right\rangle=\left\langle\hat{\psi}_{m},-\mathrm{i} \omega_{n} \hat{\psi}_{n}^{\dagger}\right\rangle$,

$\mathrm{i} \omega_{m}^{*}\left\langle\hat{\psi}_{m}, \hat{\psi}_{n}^{\dagger}\right\rangle=-\mathrm{i} \omega_{n}\left\langle\hat{\psi}_{m}, \hat{\psi}_{n}^{\dagger}\right\rangle$

$\left(\omega_{m}^{*}+\omega_{n}\right)\left\langle\hat{\psi}_{m}, \hat{\psi}_{n}^{\dagger}\right\rangle=0$.

This is the bi-orthogonality condition: every adjoint mode is orthogonal to every direct mode, except for the pairs that satisfy $\omega_{n}=-\omega_{m}^{*}$. 


\subsection{Structural sensitivity}

We would like to find the change in the direct eigenvalue, $\delta \omega_{m}$, when there is a small change, $\delta \mathrm{L}$, in the direct linear operator, $\mathrm{L}$ :

$\delta \omega_{m}=\lim _{\epsilon \rightarrow 0}\left(\frac{\omega_{m}(\mathrm{~L}+\epsilon \delta \mathrm{L})-\omega_{m}(\mathrm{~L})}{\epsilon}\right)$.

This perturbation causes perturbed eigenvalues, $\omega_{m}+\epsilon \delta \omega_{m}$, perturbed direct eigenmodes, $\hat{\psi}_{m}+\epsilon \delta \hat{\psi}_{m}$, and perturbed adjoint eigenmodes, $\hat{\psi}_{n}^{\dagger}+\epsilon \delta \hat{\psi}_{n}^{\dagger}$. We premultiply (5) by $\hat{\psi}_{n}^{\dagger}$ and substitute in the perturbed variables:

$$
\begin{aligned}
& \left\langle\left(\hat{\psi}_{n}^{\dagger}+\epsilon \delta \hat{\psi}_{n}^{\dagger}\right),\left(\mathrm{i} \omega_{m}+\mathrm{i} \epsilon \delta \omega_{m}\right)\left(\hat{\psi}_{m}+\epsilon \delta \hat{\psi}_{m}\right)\right\rangle \\
& \quad+\left\langle\left(\hat{\psi}_{n}^{\dagger}+\epsilon \delta \hat{\psi}_{n}^{\dagger}\right),(\mathrm{L}+\epsilon \delta \mathrm{L})\left(\hat{\psi}_{m}+\epsilon \delta \hat{\psi}_{m}\right)\right\rangle=0 .
\end{aligned}
$$

Retaining terms at order $\epsilon$ and making use of (5), (6), and the biorthogonality condition (12) leads to

$\delta \omega_{m}=\mathrm{i} \frac{\left\langle\hat{\psi}_{m}^{\dagger}, \delta \mathrm{L} \hat{\psi}_{m}\right\rangle}{\left\langle\hat{\psi}_{m}^{\dagger}, \hat{\psi}_{m}\right\rangle}$.

This is the penultimate term in Eq. (9) of [2], but expressed in the notation of this paper. The operator $\delta \mathrm{L}$ describes a generic perturbation to the operator, L. If one considers a perturbation that is localized in space then the structural sensitivity [6, Section 8] is defined as:

$\nabla_{L} \omega_{m} \equiv \mathrm{i} \frac{\hat{\psi}_{m}^{*} \hat{\psi}_{m}^{\dagger}}{\left\langle\hat{\psi}_{m}^{\dagger}, \hat{\psi}_{m}\right\rangle}$,

where the numerator is a function of $x$ and the eigenfunctions are usually normalized such that the denominator is 1 . This is shown graphically in [2, Fig 5 a,b].

\section{Local analysis of the direct and adjoint Ginzburg-Landau equations}

For the Ginzburg-Landau (G-L) equation, the operator L acting on $\psi(x, t)$ in (1) is:

$\frac{\partial \psi}{\partial t}=\mathrm{L} \psi \equiv a_{0}(x) \psi+a_{1}(x) \frac{\partial \psi}{\partial x}+a_{2}(x) \frac{\partial^{2} \psi}{\partial x^{2}}$,

where $a_{0}, a_{1}$ and $a_{2}$ are complex coefficients that depend on the spatial coordinate, $x$. The aim of this section is to perform WKBJ analysis on the direct and adjoint $\mathrm{G}-\mathrm{L}$ equations in order to determine $\omega_{n}^{\dagger}$ in terms of $\omega_{m}$ and $k_{n}^{\dagger}$ in terms of $k_{m}$, and to confirm that higher-order terms in the WKBJ analysis do not need to be considered. In this section, the subscripts $m$ and $n$ will be dropped because the adjoint mode constructed in Section 3.6 is always the bi-orthogonal counterpart of the direct mode constructed in Section 3.5 .

\subsection{Local dispersion relation of the direct $G-L$ equation}

In slowly-evolving flows, the coefficients $a_{0}, a_{1}$ and $a_{2}$ in (17) depend only on a slow spatial coordinate $X=\epsilon X$. The small parameter $\epsilon \ll 1$ measures the ratio between typical instability and typical inhomogeneity length scales. Implementing a WKBJ analysis, a global-mode solution of (17) is sought in the form

$\psi \sim A(X) \exp \left(\frac{\mathrm{i}}{\epsilon} \int^{X} k(u) \mathrm{d} u-\mathrm{i} \omega t\right)$,

where the local complex wavenumber $k(X)$ is a solution of the local dispersion relation:

$\omega=\Omega(k, X) \equiv \mathrm{i} a_{0}(X)-a_{1}(X) k-\mathrm{i} a_{2}(X) k^{2}$.
The dispersion relation can also be written in terms of the local absolute frequency, $\omega_{0}(X)$, the local absolute wavenumber, $k_{0}(X)$, and the local curvature, $\omega_{k k}(X)$ :

$\Omega(k, X)=\omega_{0}(X)+\frac{1}{2} \omega_{k k}(X)\left(k-k_{0}(X)\right)^{2}$,

where $\omega_{0}=\mathrm{i} a_{0}-\mathrm{i} a_{1}^{2} / 4 a_{2}, k_{0}=\mathrm{i} a_{1} / 2 a_{2}$, and $\omega_{k k}=-2 \mathrm{i} a_{2}$. (Equivalently, $a_{0}=-\mathrm{i} \omega_{0}-\mathrm{i} \omega_{k k} k_{0}^{2} / 2, a_{1}=\omega_{k k} k_{0}$, and $a_{2}=\mathrm{i} \omega_{k k} / 2$.) This shows how the coefficients of the $\mathrm{G}$-L equation can be derived from the dispersion relation associated with a given weakly developing shear flow by taking a Taylor expansion around the saddle point, which is at $\left(\omega_{0}, k_{0}\right)$ and by definition has $\mathrm{d} \omega / \mathrm{d} k=0$. Eq. (20) can be rearranged to give $k$ as an explicit function of $\omega$ :

$k^{ \pm}(X, \omega)=k_{0}(X) \pm \sqrt{2 \frac{\omega-\omega_{0}(X)}{\omega_{k k}(X)}}$.

Here, branch cuts of (21) are taken along positive real values of the argument of the square root. This choice of branch cut ensures that, in stable or convectively unstable regions of the complex $X$-plane, the above definition coincides with the usual labelling of spatial branches based on causality considerations, for which a $k^{+}$-branch corresponds to a downstream response to localized harmonic forcing, and a $k^{-}$-branch corresponds to an upstream response.

\subsection{Calculation of the adjoint of the G-L equation}

For the G-L equation, the adjoint operator, $\mathrm{L}^{\dagger}$, is found by expanding $\left\langle\hat{\psi}^{\dagger}, \mathrm{L} \hat{\psi}\right\rangle$, using (7), and then integrating by parts:

$$
\begin{aligned}
\left\langle\hat{\psi}^{\dagger}, \mathrm{L} \hat{\psi}\right\rangle= & \int_{-\infty}^{+\infty} \hat{\psi}^{\dagger *}\left(a_{0} \hat{\psi}+a_{1} \frac{\partial \hat{\psi}}{\partial x}+a_{2} \frac{\partial^{2} \hat{\psi}}{\partial x^{2}}\right) \mathrm{d} x \\
= & \int_{-\infty}^{+\infty}\left(a_{0} \hat{\psi}^{\dagger *}-\frac{\partial}{\partial x}\left(a_{1} \hat{\psi}^{\dagger *}\right)+\frac{\partial^{2}}{\partial x^{2}}\left(a_{2} \hat{\psi}^{\dagger *}\right)\right) \\
& \times \hat{\psi} \mathrm{d} x,
\end{aligned}
$$

in which the boundary terms have been set to zero with appropriate boundary conditions. The adjoint operator is found by noting that, from (8), $\left\langle\hat{\psi}^{\dagger}, \mathrm{L} \hat{\psi}\right\rangle=\left\langle\mathrm{L}^{\dagger} \hat{\psi}^{\dagger}, \hat{\psi}\right\rangle$, and therefore that

$$
\begin{aligned}
\mathrm{L}^{\dagger} \hat{\psi}^{\dagger} & =a_{0}^{*} \hat{\psi}^{\dagger}-\frac{\partial}{\partial x}\left(a_{1}^{*} \hat{\psi}^{\dagger}\right)+\frac{\partial^{2}}{\partial x^{2}}\left(a_{2}^{*} \hat{\psi}^{\dagger}\right) \\
& =a_{0}^{\dagger} \hat{\psi}^{\dagger}+a_{1}^{\dagger} \frac{\partial \hat{\psi}^{\dagger}}{\partial x}+a_{2}^{\dagger} \frac{\partial^{2} \hat{\psi}^{\dagger}}{\partial x^{2}},
\end{aligned}
$$

where $a_{0}^{\dagger} \equiv a_{0}^{*}-\partial a_{1}^{*} / \partial x+\partial^{2} a_{2}^{*} / \partial x^{2}, a_{1}^{\dagger} \equiv-a_{1}^{*}+2 \partial a_{2}^{*} / \partial x$, and $a_{2}^{\dagger} \equiv a_{2}^{*}$. These expressions are general and do not necessarily assume weak spatial inhomogeneities.

\subsection{Local dispersion relation of the adjoint problem}

Under the quasi-parallel-flow assumption, the coefficients of the direct $\mathrm{G}-\mathrm{L}$ equations depend only on the slow spatial coordinate $X=\epsilon X$. Eq. (24) becomes:

$$
\begin{aligned}
\mathrm{L}^{\dagger} \hat{\psi}^{\dagger}= & a_{0}^{*} \hat{\psi}^{\dagger}-a_{1}^{*} \frac{\partial \hat{\psi}^{\dagger}}{\partial x}-\hat{\psi}^{\dagger} \epsilon \frac{\partial a_{1}^{*}}{\partial X} \\
& +a_{2}^{*} \frac{\partial^{2} \hat{\psi}^{\dagger}}{\partial x^{2}}+2 \epsilon \frac{\partial a_{2}^{*}}{\partial X} \frac{\partial \hat{\psi}^{\dagger}}{\partial x}+\hat{\psi}^{\dagger} \epsilon^{2} \frac{\partial^{2} a_{2}^{*}}{\partial X^{2}} .
\end{aligned}
$$

When performing a WKBJ analysis of the adjoint G-L equation (2), the adjoint operator (24) must be expanded in powers of $\epsilon$ as

$$
\begin{aligned}
\mathrm{L}^{\dagger}\left(\frac{\partial}{\partial X} ; X\right)= & \mathrm{L}_{0}^{\dagger}\left(\frac{\partial}{\partial X} ; X\right) \\
& +\epsilon \mathrm{L}_{1}^{\dagger}\left(\frac{\partial}{\partial X} ; X\right)+\epsilon^{2} \mathrm{~L}_{2}^{\dagger}\left(\frac{\partial}{\partial X} ; X\right),
\end{aligned}
$$


By inspection of (26), $\mathrm{L}_{0}^{\dagger}, \mathrm{L}_{1}^{\dagger}$, and $\mathrm{L}_{2}^{\dagger}$ are:

$\mathrm{L}_{0}^{\dagger}\left(\frac{\partial}{\partial X} ; X\right)=a_{0}^{*}(X)-a_{1}^{*}(X) \frac{\partial}{\partial x}+a_{2}^{*}(X) \frac{\partial^{2}}{\partial x^{2}}$,

$\mathrm{L}_{1}^{\dagger}\left(\frac{\partial}{\partial X} ; X\right)=-\frac{\partial a_{1}^{*}(X)}{\partial X}+2 \frac{\partial a_{2}^{*}(X)}{\partial X} \frac{\partial}{\partial x}$,

$\mathrm{L}_{2}^{\dagger}\left(\frac{\partial}{\partial X} ; X\right)=\frac{\partial^{2} a_{2}^{*}(X)}{\partial X^{2}}$.

A solution of the adjoint problem is then sought in the form

$$
\begin{aligned}
\psi^{\dagger} \sim & \left(A_{0}^{\dagger}(X)+\epsilon A_{1}^{\dagger}(X)+\epsilon^{2} A_{2}^{\dagger}(X)+\cdots\right) \\
& \times \exp \left(\frac{\mathrm{i}}{\epsilon} \int^{X} k(u) \mathrm{d} u-\mathrm{i} \omega^{\dagger} t\right) .
\end{aligned}
$$

Substituting (28)-(31) into the governing adjoint equation (2) gives, at leading-order,

$$
-\mathrm{i} \omega^{\dagger}=a_{0}^{*}(X)-a_{1}^{*}(X) \mathrm{i} k(X)-a_{2}^{*}(X) k^{2}(X)=\mathrm{L}_{0}^{\dagger}(\mathrm{i} k ; X) .
$$

In a manner similar to the direct problem, the adjoint dispersion relation can be rewritten as

$$
\begin{aligned}
\omega^{\dagger} & =\Omega_{0}^{\dagger}(k, X) \equiv \mathrm{i} a_{0}^{*}(X)+a_{1}^{*}(X) k(X)-\mathrm{i} a_{2}^{*}(X) k^{2}(X) \\
& =\omega_{0}^{\dagger}(X)+\frac{1}{2} \omega_{k k}^{\dagger}(X)\left(k-k_{0}^{\dagger}(X)\right)^{2},
\end{aligned}
$$

where

$$
\begin{aligned}
& \omega_{0}^{\dagger}(X)=\mathrm{i} a_{0}^{*}(X)-\frac{\mathrm{i}}{4} a_{1}^{* 2}(X) / a_{2}^{*}(X)=-\omega_{0}^{*}(X), \\
& k_{0}^{\dagger}(X)=-\frac{\mathrm{i}}{2} a_{1}^{*}(X) / a_{2}^{*}(X)=k_{0}^{*}(X), \\
& \omega_{k k}^{\dagger}(X)=-2 \mathrm{i} a_{2}^{*}(X)=-\omega_{k k}^{*}(X) .
\end{aligned}
$$

The higher-order terms $\mathrm{L}_{1}^{\dagger}$ and $\mathrm{L}_{2}^{\dagger}$ do not appear in this adjoint dispersion relation, because it is obtained at leading order in the WKBJ analysis. The $\mathrm{L}_{1}^{\dagger}$ component enters only when working out, at $O\left(\epsilon^{1}\right)$, the solvability condition that governs the leading-order amplitude term $A_{0}^{\dagger}(X)$ in (31). This amplitude equation is

$$
\begin{aligned}
& \Omega_{0, k}^{\dagger}(k(X), X) \frac{\mathrm{d} A_{0}^{\dagger}}{\mathrm{d} X}+\frac{1}{2} \Omega_{0, k k}^{\dagger}(k(X), X) \frac{\mathrm{d} k}{\mathrm{~d} X} A_{0}^{\dagger}(X) \\
& +\mathrm{i} \Omega_{1}^{\dagger}(k(X), X) A_{0}^{\dagger}(X)=0,
\end{aligned}
$$

where $\Omega_{1}^{\dagger}(k, X) \equiv \mathrm{iL}_{1}^{\dagger}(\mathrm{i} k, X)$. Higher-order expansions will not be derived further, however, because the results of this paper require only the local dispersion relations. Turning points, where $\partial \Omega_{0}^{\dagger} / \partial k=0$, are not affected by the higher-order expansions.

The key point of this section is that, at leading order, the dispersion relation of the adjoint $\mathrm{G}$-L equation is the same as that of the direct G-L equation but with the substitutions (35)-(37).

\subsection{Adjoint of a generic polynomial PDE}

The development in Sections 3.1-3.3 is for a parabolic PDE but holds for any polynomial PDE in one spatial dimension, as shown in this section. For a generic polynomial PDE, the direct operator (17) can be written as

$$
\frac{\partial \psi}{\partial t}=\mathrm{L} \psi \equiv \sum_{j} a_{j}(x) \frac{\partial^{j} \psi}{\partial x^{j}},
$$

and, after integration by parts, the adjoint operator can be written as

$$
\frac{\partial \psi^{\dagger}}{\partial t}=\mathrm{L}^{\dagger} \psi^{\dagger} \equiv \sum_{j}(-1)^{j} \frac{\partial^{j}}{\partial x^{j}}\left(a_{j}^{*}(x) \psi^{\dagger}\right) .
$$

If the coefficients $a_{j}^{*}$ do not depend on $x$ then $L^{\dagger} \psi^{\dagger}=\sum_{j}(-1)^{j} a_{j}^{*}$ $\partial_{x}^{j} \psi^{\dagger}$ and (35)-(37) follow immediately. If the coefficients $a_{j}^{*}$ depend on $x$, then the $x$-derivatives of $a_{j}^{*}(x) \psi^{\dagger}$ produce extra terms:

$$
\begin{aligned}
\mathrm{L}^{\dagger} \psi^{\dagger}= & \left(a_{0}^{*}(x)-\frac{\partial a_{1}^{*}(x)}{\partial x}+\frac{\partial^{2} a_{2}^{*}(x)}{\partial x^{2}}-\frac{\partial^{3} a_{3}^{*}(x)}{\partial x^{3}}+\cdots\right) \psi^{\dagger} \\
& +\left(-a_{1}^{*}(x)+2 \frac{\partial a_{2}^{*}(x)}{\partial x}-3 \frac{\partial^{2} a_{3}^{*}(x)}{\partial x^{2}}+\cdots\right) \frac{\partial \psi^{\dagger}}{\partial x} \\
& +\left(a_{2}^{*}(x)-3 \frac{\partial a_{3}^{*}(x)}{\partial x}+\cdots\right) \frac{\partial^{2} \psi^{\dagger}}{\partial x^{2}} \\
& +\left(-a_{3}^{*}(x)+\cdots\right) \frac{\partial^{3} \psi^{\dagger}}{\partial x^{3}}+\cdots
\end{aligned}
$$

However, under the assumption of slow spatial development, the $n^{\text {th }}$ derivatives of the coefficients $a_{j}$ are of order $\epsilon^{n}$, so the local dispersion relation that is obtained at leading order is the same as that obtained for constant coefficients. This proves that the relations $\omega_{0}^{\dagger}(X)=-\omega_{0}^{\star}(X), k_{0}^{\dagger}(X)=k_{0}^{\star}(X)$ and $\omega_{k k}^{\dagger}(X)=-\omega_{k k}^{\star}(X)$ in (35)-(37) hold for systems governed by any dispersion relation that is polynomial in $k$. We therefore expect this result to remain generally valid in the case of dispersion relations that are analytic in $k$ over large parts of the complex $k$-plane. We assume that dispersion relations derived from the linearized Navier-Stokes equations in slowly-varying flows fall into this category.

\subsection{Global mode of the direct $G-L$ equation with a local analysis}

A linear global mode is a global solution of the governing equation (1) with the form $\psi(x, t) \sim \exp \left(-\mathrm{i} \omega_{g} t\right)$ for a complex global frequency $\omega_{g}$. Assuming that the slowly-varying coefficients $\omega_{0}(X), k_{0}(X)$ and $\omega_{k k}(X)$ are known along the real $X$-axis, a WKBJ approximation of the global mode can be sought as in (18) with $\omega=\omega_{g}$. This integral is most easily evaluated in the complex $X$-plane, as shown in the top half of Fig. 1a. (The bottom half is for the adjoint mode.) The point $X_{s}$ is a saddle point of $\omega_{0}(X)$ and the diagonal lines have the same value of $\omega_{0 i}$ as the saddle point. Huerre and Monkewitz [19] have shown that the frequency of the dominant global mode, $\omega_{g}$, is equal to $\omega_{s}+O(\epsilon)$, where $\omega_{s}=\omega_{0}\left(X_{s}\right)$.

At a given $\omega_{g}$, there are two valid solutions to $k$, known as the $k^{+}$ and $k^{-}$spatial branches, and there are therefore two independent WKBJ approximations

$\psi^{+} \sim A^{+}(X) \exp \left(\frac{\mathrm{i}}{\epsilon} \int_{X_{S}}^{X} k^{+}\left(u, \omega_{g}\right) \mathrm{d} u-\mathrm{i} \omega_{g} t\right)$

and

$\psi^{-} \sim A^{-}(X) \exp \left(\frac{\mathrm{i}}{\epsilon} \int_{X_{S}}^{X} k^{-}\left(u, \omega_{g}\right) \mathrm{d} u-\mathrm{i} \omega_{g} t\right)$.

These two WKBJ approximations are singular at the saddle point $X_{s}$, which is a double turning point of the dispersion relation. From this double turning point, four Stokes lines emerge, defined by

$\operatorname{Im} \int_{X_{S}}^{X}\left[k^{+}\left(u, \omega_{s}\right)-k^{-}\left(u, \omega_{s}\right)\right] \mathrm{d} u=0$.

Along these Stokes lines both WKBJ approximations remain of the same order of magnitude, while inside the sectors delimited by 

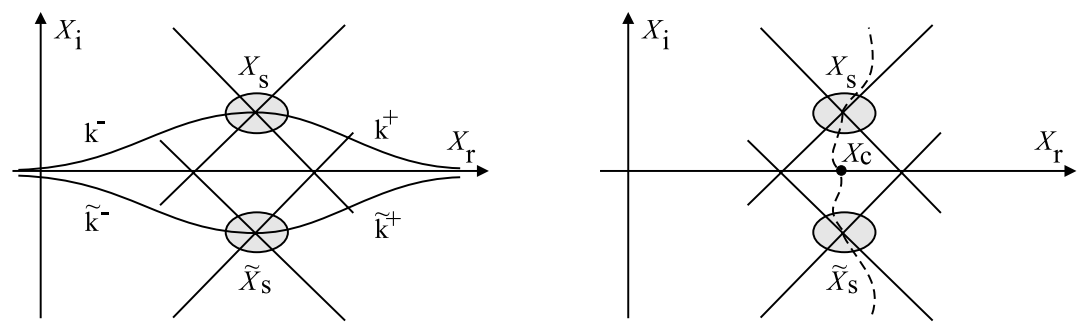

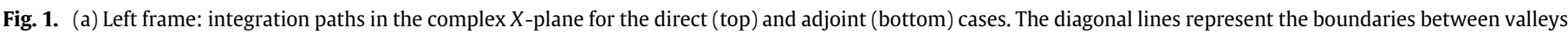

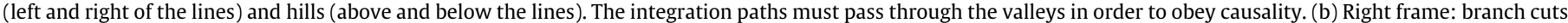
(dashed lines) in the complex $X$-plane.

the Stokes lines one approximation is exponentially larger than the other. Following classical WKBJ theory [25] the global mode must be sought as a linear combination of the two independent solutions, $\psi=C^{+} \psi^{+}+C^{-} \psi^{-}$, within each sector delimited by these Stokes lines.

When $X \rightarrow+\infty$, the solution must be dominated by a $k^{+}$ branch and is therefore made up of the subdominant $\psi^{+}$approximation. The global mode is therefore of the form $\psi=C^{+} \psi^{+}$(with $C^{-}=0$ ) in the region starting from the Stokes lines issuing from $X_{s}$ and extending to $X=+\infty$. (See Pier [26] for a detailed analysis of a similar case.) For similar reasons, the global mode is of the form $\psi=C^{-} \psi^{-}$(with $C^{+}=0$ ) in the region starting from the Stokes lines issuing from $X_{s}$ and extending to $X=-\infty$.

Consequently, the global mode is approximated by the WKBJ approximation $C^{-} \psi^{-}$along the semi-infinite path from $X_{s}$ to $-\infty$, and by $C^{+} \psi^{+}$along the semi-infinite path from $X_{s}$ to $+\infty$. Since the global mode must be continuous at $X_{s}$, the coefficient $C^{+}$on the path from $X_{s}$ to $+\infty$ must equal $C^{-}$on the path from $X_{s}$ to $-\infty$ (this includes higher-order terms; asymptotic matching of the two WKBJ-expansions prevailing on each side of the saddle point can be rigorously carried out via an inner layer). After rescaling the solution so that $C^{+}=C^{-}=1$, the direct global mode is approximated by $\psi^{+}$along the semi-infinite path from $X_{s}$ to $X=+\infty$ and by $\psi^{-}$ along the semi-infinite path from $X_{s}$ to $X=-\infty$.

Finally, the approximations of the direct global mode obtained along the path from $X=-\infty$ to $X=+\infty$, passing through the saddle point $X_{S}$, must be continued onto the real $X$-axis. When crossing a Stokes line, a subdominant WKBJ solution becomes dominant but remains a valid asymptotic approximation. Therefore the global mode is approximated by $\psi^{-}$in the sectors adjacent to the sector extending to $X=-\infty$, and by $\psi^{+}$in the sectors adjacent to the sector extending to $X=+\infty$. Since there are four Stokes lines and two branch cuts emanating from the saddle point, $X_{s}$, one may safely assume that one branch cut crosses the real axis at $X_{c}$ and that no more than one Stokes line crosses the real axis on either side of $X_{c}$. It follows that the global mode is approximated by $\psi^{-}$for $X<X_{c}$ along the real axis and by $\psi^{+}$for $X>X_{c}$. At $X_{c}$ there is a smooth relabelling of the $k$-branches, but otherwise nothing special happens across the branch cut. This division of the integration path becomes important in Section 3.7.

\subsection{Global mode of the adjoint $G$-L equation with a local analysis}

Following the same development as Section 3.5, the adjoint global mode is sought as

$\psi^{\dagger} \sim A^{\dagger}(X) \exp \left[\frac{\mathrm{i}}{\epsilon} \int^{X} k^{\dagger}\left(u ; \omega_{g}^{\dagger}\right) \mathrm{d} u-\mathrm{i} \omega_{g}^{\dagger} t\right]$.

We again assume that the coefficients $\omega_{0}(X), k_{0}(X)$ and $\omega_{k k}(X)$ can be continued analytically into the complex plane and use the relationships (35)-(37). This is represented in the bottom half of Fig. 1(a). We obtain the result that $\omega_{g}^{\dagger}=\omega_{s}^{\dagger}+O(\epsilon)$, where $\omega_{s}^{\dagger}=$
$\omega_{0}^{\dagger}\left(X_{s}^{\dagger}\right)$ with $\mathrm{d} \omega_{0}^{\dagger} /\left.\mathrm{d} X\right|_{X_{s}^{\dagger}}=0$. The local wavenumber in (48) follows the $k^{\dagger-}$ branch for $X \rightarrow-\infty$ and the $k^{\dagger+}$ branch for $X \rightarrow+\infty$. Here, these branches are obtained from the local adjoint dispersion relation (36) as

$k^{\dagger \pm}\left(X, \omega_{g}^{\dagger}\right)=k_{0}^{\dagger}(X) \pm \sqrt{2 \frac{\omega_{g}^{\dagger}-\omega_{0}^{\dagger}(X)}{\omega_{k k}^{\dagger}(X)}}$.

For real values of $X$, substituting (35)-(37) into (49) leads to the following relationship between the local branches of the adjoint and the direct global modes:

$k^{\dagger \pm}\left(X ; \omega_{g}^{\dagger}\right)=\left(k^{\mp}\left(X ; \omega_{g}\right)\right)^{*}$

This relationship guarantees that a branch cut of the adjoint $k^{\dagger^{ \pm}}$ crosses the real $X$-axis at the same location, $X_{c}$, as a branch cut of the direct $k^{ \pm}$.

The two adjoint spatial branches $k^{\dagger \pm}$ lead to two independent WKBJ approximations

$\psi^{\dagger^{+}} \sim A^{\dagger^{+}}(X) \exp \left(\frac{\mathrm{i}}{\epsilon} \int_{X_{s}^{\dagger}}^{X} k^{\dagger^{+}}\left(u, \omega_{g}^{\dagger}\right) \mathrm{d} u-\mathrm{i} \omega_{g}^{\dagger} t\right)$

and

$\psi^{\dagger^{-}} \sim A^{\dagger^{-}}(X) \exp \left(\frac{\mathrm{i}}{\epsilon} \int_{X_{s}^{\dagger}}^{X} k^{\dagger^{-}}\left(u, \omega_{g}^{\dagger}\right) \mathrm{d} u-\mathrm{i} \omega_{g}^{\dagger} t\right)$.

Following similar arguments to those in the previous section, it can be shown that the adjoint global mode is approximated along the real axis by $\psi^{\dagger^{-}}$for $X<X_{c}$ and by $\psi^{\dagger^{+}}$for $X>X_{c}$. For $X<X_{c}$, the direct global mode follows $k^{-}$and the adjoint global mode follows $k^{\dagger}$, which is $\left(k^{+}\right)^{*}$. For $X>X_{c}$, the direct global mode follows $k^{+}$and the adjoint global mode follows $k^{\dagger}$, which is $\left(k^{-}\right)^{*}$. At $X_{c}$, there is a smooth re-labelling of the $k$-branches.

The final result, that the adjoint mode follows $\left(k^{+}\right)^{*}$ upstream of the wavemaker and $\left(k^{-}\right)^{*}$ downstream, is simple and may seem trivial. However, we are not aware of this result being stated or used before in stability analysis, despite its potential usefulness.

\subsection{Calculating the structural sensitivity of the $G-L$ equation with a local analysis}

The structural sensitivity (16) is the product of the direct and adjoint global modes. For $X$ along the real axis, the direct global mode found from the local analysis takes the form

$\psi \sim \begin{cases}A^{+}(X) \exp \left[\frac{\mathrm{i}}{\epsilon} \int_{X_{S}}^{X} k^{+} \mathrm{d} u\right] & \text { for } X>X_{c}, \\ A^{-}(X) \exp \left[\frac{\mathrm{i}}{\epsilon} \int_{X_{S}}^{X} k^{-} \mathrm{d} u\right] & \text { for } X<X_{c} .\end{cases}$

After splitting the integrals from $X_{S}$ to $X$ into two integrals from $X_{S}$ to $X_{c}$ and from $X_{c}$ to $X$, and using the fact that the $k^{+}$-branch on the 
right of the branch cut is identical to the $k^{-}$-branch on the left of the branch cut, the linear global mode may be renormalized as

$\psi \sim \begin{cases}A^{+}(X) \exp \left[\frac{\mathrm{i}}{\epsilon} \int_{X_{c}}^{X} k^{+} \mathrm{d} u\right] & \text { for } X>X_{c}, \\ A^{-}(X) \exp \left[\frac{\mathrm{i}}{\epsilon} \int_{X_{c}}^{X} k^{-} \mathrm{d} u\right] & \text { for } X<X_{c} .\end{cases}$

Similarly, the adjoint global mode found from the local analysis takes the following form along the real axis

$\psi^{\dagger} \sim \begin{cases}A^{\dagger^{+}}(X) \exp \left[\frac{\mathrm{i}}{\epsilon} \int_{X_{c}}^{X} k^{\dagger^{+}} \mathrm{d} u\right] & \text { for } X>X_{c}, \\ A^{\dagger^{-}}(X) \exp \left[\frac{\mathrm{i}}{\epsilon} \int_{X_{c}}^{X} k^{\dagger^{-}} \mathrm{d} u\right] & \text { for } X<X_{c} .\end{cases}$

With the identity (50), it follows that

$\psi^{\dagger} \sim \begin{cases}A^{\dagger^{+}}(X) \exp \left[\frac{\mathrm{i}}{\epsilon} \int_{X_{C}}^{X} k^{-*} \mathrm{~d} u\right] & \text { for } X>X_{c}, \\ A^{\dagger^{-}}(X) \exp \left[\frac{\mathrm{i}}{\epsilon} \int_{X_{C}}^{X} k^{+*} \mathrm{~d} u\right] & \text { for } X<X_{c} .\end{cases}$

The structural sensitivity $\nabla_{L} \omega_{m}$ from (16) may now be obtained by computing the product $\psi^{*} \psi^{\dagger}$. Noting that $(\exp (\mathrm{i} z))^{*}=$ $\exp \left(-\mathrm{i} z^{*}\right)$ yields

$\nabla_{L} \omega_{m} \propto\left\{\begin{array}{l}A^{\dagger^{+}}(X)\left[A^{+}(X)\right]^{*} \exp \left[\frac{\mathrm{i}}{\epsilon} \int_{X_{c}}^{X}\left(k^{-}-k^{+}\right)^{*} \mathrm{~d} u\right] \\ \text { for } X>X_{c}, \\ A^{\dagger^{-}}(X)\left[A^{-}(X)\right]^{*} \exp \left[\frac{\mathrm{i}}{\epsilon} \int_{X_{c}}^{X}\left(k^{+}-k^{-}\right)^{*} \mathrm{~d} u\right] \\ \text { for } X<X_{c} .\end{array}\right.$

The magnitude of the structural sensitivity is therefore obtained as

$\left|\nabla_{L} \omega_{m}\right| \propto\left\{\begin{array}{c}B(X) \exp \left[\frac{1}{\epsilon} \int_{X_{c}}^{X} \operatorname{Im}\left(k^{-}-k^{+}\right) \mathrm{d} u\right] \\ \text { for } X>X_{c}, \\ B(X) \exp \left[\frac{1}{\epsilon} \int_{X_{c}}^{X} \operatorname{Im}\left(k^{+}-k^{-}\right) \mathrm{d} u\right] \\ \text { for } X<X_{c},\end{array}\right.$

where $B(X)$ is a slowly varying coefficient.

The wavenumbers $k^{+}$and $k^{-}$are given by (21) with $\omega=\omega_{s}$. In a configuration that, when $X \rightarrow \pm \infty$, is stable or at most convectively unstable, $\operatorname{Im}\left(k^{+}-k^{-}\right)>0$ for sufficiently large $|X|$ on the real axis. Therefore the structural sensitivity necessarily decays for $X \rightarrow \pm \infty$ and the maximum structural sensitivity corresponds to the location where $\operatorname{Im}\left(k^{+}-k^{-}\right)=0$. For the G-L equation, $\operatorname{Im}\left(k^{+}-\right.$ $k^{-}$) is always positive, due to our definition of the branch cut, and vanishes only at $X_{c}$. This means that $\left|\nabla_{L} \omega_{m}\right|$ is a maximum at $X_{c}$. In general, it is true that the maximum structural sensitivity is found where $\operatorname{Im}\left(k^{+}-k^{-}\right)=0$. However, it is not necessarily located on the branch cut, because the location where $\operatorname{Im}\left(k^{+}-k^{-}\right)=0$ does not necessarily coincide with the (arbitrary) choice of branch cut.

This result, which is for flows with infinite streamwise extent, should not be confused with the Kulikovskii criterion [27, Section 65], which is for flows with finite streamwise extent. In those flows, the downstream travelling waves, $k^{+}$, reflect off the downstream boundary and the upstream travelling waves, $k^{-}$, reflect off the upstream boundary. The function describing the wave must be singly-valued between the boundaries, which means that only certain combinations of $k^{+}$and $k^{-}$are permitted. For long (but streamwise-confined) systems, this constraint reduces to $\operatorname{Im}\left(k^{+}-\right.$ $\left.k^{-}\right)=0$. In those flows, the permitted global mode frequen- cies are then calculated by combining this constraint with the local dispersion relation. For flows with finite extent, the relation $\operatorname{Im}\left(k^{+}-k^{-}\right)=0$ therefore serves as an additional constraint on the global complex frequency, $\omega_{g}$, of the flow. For flows with infinite extent, on the other hand, there is no corresponding restriction on $\omega_{g}$ and the point where $\operatorname{Im}\left(k^{+}-k^{-}\right)=0$ merely indicates the centre of the structural sensitivity.

The wavemaker in a local analysis and the structural sensitivity in a global analysis differ both in concept and in outcome. Nevertheless, the two regions lie close to each other and there is a link between the two, which can be summarized as follows. The complex frequency of the global mode, $\omega_{g}$, is the absolute frequency at the saddle point $X_{s}$ of $\omega_{0}\left(X_{s}\right)$ in the complex $X$-plane. The region around the saddle point in the complex $X$-plane is the wavemaker region in the local sense given by Huerre and Monkewitz [19]. It could be tempting to assume that the real component of $X_{s}$ has physical significance. However, this is only an approximation to the position of the maximum of the structural sensitivity (i.e. the global concept) in cases where $\operatorname{Im}\left(X_{S}\right)$ is small. Instead, to work out the position of maximum structural sensitivity from a local analysis, the spatial branches, $k^{+}$and $k^{-}$, must be calculated at the global mode frequency, $\omega_{g}$. Because $\operatorname{Im}\left(\omega_{g}\right)<\operatorname{Im}\left(\omega_{0}\right)$ along the real $X$ axis, the point of maximum structural sensitivity, in the global sense given by [6], is the point at which $\operatorname{Im}\left(k^{+}-k^{-}\right)=0$.

\section{Local analysis of the direct and adjoint Linearized Navier- Stokes equations}

The planar linearized Navier-Stokes (LNS) equations for a perturbation $\tilde{\mathbf{q}}(x, z, t) \equiv\left[\tilde{v}_{1}(x, z, t), \tilde{v}_{2}(x, z, t), \tilde{p}(x, z, t)\right]^{T}$ are expressed as three PDEs in the three primitive variables, $\left(\tilde{v}_{1}, \tilde{v}_{2}, \tilde{p}\right)$. The WKBJ analysis is performed, reducing these three PDEs to three ODEs for the Fourier/Laplace modes $\hat{\boldsymbol{q}}_{m}(z) \exp \left\{\mathrm{i}\left(k_{m} x-\omega_{m} t\right)\right\}$. These ODEs are then expressed as the generalized eigenvalue problem:

$-\mathrm{i} \omega_{m} \mathrm{~B} \hat{\boldsymbol{q}}_{m}-\mathrm{A} \hat{\boldsymbol{q}}_{m}=0$.

The problem is discretized by replacing the operators $\mathrm{A}\left(k_{m}\right)$ and $\mathrm{B}\left(k_{m}\right)$, which act on the continuous field $\hat{\boldsymbol{q}}_{m}(z)$, with matrices $A\left(k_{m}\right)$ and $B\left(k_{m}\right)$, which act on a state vector $\varphi_{m}$. This state vector holds the values of $\hat{\boldsymbol{q}}_{m}$ at $N$ gridpoints at $z_{j}, j \in[1, N]$. The local direct LNS equations (59) are thereby expressed as the generalized matrix eigenvalue problem

$-\mathrm{i} \omega_{m} B \varphi_{m}-A \varphi_{m}=0$,

which serves as the dispersion relation for the calculation of $\omega_{0}(X),\left(\omega_{s}, X_{s}\right), \omega_{g}, k^{+}(X)$ and $k^{-}(X)$. Computing the dispersion relation for the Navier-Stokes problem is technically more difficult than for the Ginzburg-Landau problem, for which it is explicit. However, once the dispersion relation is expressed numerically, the subsequent calculations and derivations of quantities such as spatial branches and local absolute frequencies are carried out in a similar manner.

The direct global mode is constructed with the technique described in Section 3.5 and [28]. In summary, the absolute complex frequency, $\omega_{0}(X)$, is calculated by finding the valid saddle point of $\omega(k)$ at each streamwise location, $X$. An $8^{\text {th }}$ order Padé polynomial is fitted to $\omega_{0}(X)$ and then extrapolated into the complex $X$ plane, as will be described in Section 5.1. The saddle point of $\omega_{0}(X)$ is identified in the complex $X$-plane and its value of $\omega_{0}$ gives the global mode complex frequency, $\omega_{g}$. Then, at the streamwise location of the saddle point in the $X$-plane, two values of $k\left(\omega_{g}\right)$ are found on either side of the saddle point $\omega_{0}$ in the $k$-plane. These are labelled $k^{+}$and $k^{-}$and they are followed upstream and downstream from this point. The local values of $k^{+}(X)$ and $k^{-}(X)$ are then integrated according to (54) in order to obtain the amplitude 
and phase of the global mode in the $X$-direction. The eigenfunctions of $k^{+}(X)$ and $k^{-}(X)$ are required in order to obtain the $z$ dependence of the global mode. At this point there is an apparent contradiction: the local eigenfunctions can be multiplied by any arbitrary constant, yet the amplitude and phase of the global mode in the $X$-direction should be dictated by (54). To avoid this contradiction, the eigenfunctions must be normalized consistently. This is analogous to the normalization required when handling the Parabolized Stability Equation [29, Eq. (9a,b)]. In this paper, the eigenfunctions are normalized such that the $v_{2}$-eigenfunctions have the same amplitude and phase at $z=0$. This is chosen because, for the sinuous perturbations considered here, the $v_{2}$-eigenfunction always has a large absolute value at $z=0$. In principle, any value of $z$ could be chosen, and for flows that are nearly parallel, such as that in Section 5.1, the choice of $z$ has only a small effect on the predicted shapes of the direct and adjoint global modes.

The adjoint global mode is calculated using the substitution derived in Section 3.3 for a parabolic dispersion relation and in Section 3.4 for a generic polynomial PDE: $\omega_{g}^{\dagger}=-\omega_{g}^{*}, k^{\dagger^{+}}=\left(k^{-}\right)^{*}$ and $k^{\dagger^{-}}=\left(k^{+}\right)^{*}$. This substitution requires the base flow to vary slowly in the streamwise direction. In this paper, this result has been shown for a generic PDE with one spatial dimension and it can be generalized (after lengthy developments) to a PDE with two spatial dimensions. For the $z$-dependence, however, the eigenfunctions of $\mathrm{k}^{+}$and $\mathrm{k}^{\dagger^{-}}$are not the same as those of $k^{-}$ and $k^{+}$, and need to be calculated from the discrete adjoint of the LNS equations. To do this, the generalized matrix eigenvalue problem (60) is post-multiplied by the adjoint eigenfunction $\varphi_{n}^{\dagger}$ and re-arranged using the discretized version of the inner product: $\left\langle\varphi_{m}, \varphi_{n}^{\dagger}\right\rangle \equiv \varphi_{m}^{H} M \varphi_{n}^{\dagger}$, where ${ }^{H}$ denotes the Hermitian transpose and $M$ is the mass matrix, whose diagonal elements are the volume of space attributed to each gridpoint:

$-\mathrm{i} \omega_{m} B \varphi_{m}-A \varphi_{m}=0$,

$\left\langle-\mathrm{i} \omega_{m} B \varphi_{m}, \varphi_{n}^{\dagger}\right\rangle-\left\langle A \varphi_{m}, \varphi_{n}^{\dagger}\right\rangle=0$,

$\left\langle\varphi_{m}, M^{-1}\left(\mathrm{i} \omega_{m}^{*}\right) B^{H} M \varphi_{n}^{\dagger}\right\rangle-\left\langle\varphi_{m}, M^{-1} A^{H} M \varphi_{n}^{\dagger}\right\rangle=0$,

$\mathrm{i} \omega_{m}^{*}\left(M^{-1} B^{H} M\right) \varphi_{n}^{\dagger}-\left(M^{-1} A^{H} M\right) \varphi_{n}^{\dagger}=0$.

The local adjoint LNS equations are written as

$-\mathrm{i} \omega_{n} B^{\dagger} \varphi_{n}^{\dagger}-A^{\dagger} \varphi_{n}^{\dagger}=0$,

so, by comparing (61) and (62),

$\omega_{n}=-\omega_{m}^{*}$,

$A^{\dagger}=M^{-1} A^{H} M$,

$B^{\dagger}=M^{-1} B^{H} M$,

and it can be shown that the bi-orthogonality condition becomes $\left(\omega_{n}-\omega_{m}^{*}\right) \varphi_{m}^{H} B^{H} M \varphi_{n}^{\dagger}=0$. The adjoint eigenvalue, $\omega_{n}$, is known from (63), so there is no need to solve (62) as a generalized eigenvalue problem. The fastest method is to calculate the adjoint matrices of the discretized problem with (64)-(65) and then to find the null space of $-\mathrm{i} \omega_{n} B^{\dagger}-A^{\dagger}$ with a $\mathrm{QR}$ decomposition.

\section{Demonstrations}

\subsection{Slowly-developing confined wake flow}

We test the procedure described in Section 4 on a slowlydeveloping flow, using the planar linearized Navier-Stokes equations. Fig. 2(a) shows the streamlines and vorticity of a confined co-flow wake at $R e=400$, with perfect slip at the top and bottom boundaries. The flow is identical to that in [28], except that it has a sharper inlet velocity profile, which makes it slightly more unstable. It is similar to the flows studied by Tammisola [17].

Fig. 2(b) shows the absolute growth rate $\omega_{0 i}(X)$. This is calculated at each axial station, $X$, by finding saddle points of $\omega(k)$ in the complex $k$-plane, using the dispersion relation formed from the matrix eigenvalue problem (60). This flow has a recirculation bubble between $2.26<X<22.42$ and is absolutely unstable over the slightly wider range of $0.05<X<28.70$.

In order to find the complex frequency of the linear global mode, $\omega_{g}$, the saddle point of $\omega_{0}(X)$ must be found in the complex $X$ plane. Its position is labelled $\left(\omega_{s}, X_{s}\right)$. For the $\mathrm{G}-\mathrm{L}$ equation (17), the coefficients were expressed in terms of this saddle point position via (20), but for the LNS equation there is no such analytical solution. Instead, 8th order Padé polynomials are fitted to $\omega_{0}(X)$ using the procedure described in [28]. Saddle points of this polynomial are then found in the complex plane, as shown in Fig. 2(c). There are several saddle points but the main one is easy to identify because it lies close to the real $X$-axis and moves very little as the order of the polynomials increases. The range of $\omega_{0}(X)$ over which the points are fitted must encompass the peak of $\omega_{0}(X)$ but is otherwise arbitrary. We performed eight calculations, fitting between all points that satisfied $\omega_{0 i}>0.15,0.10,0.05,0.00,-0.05$, $-0.10,-0.25,-0.20$ and found that $\omega_{s}$ varied by less than $1 \%$ between all these calculations.

For this flow, the polynomial is fitted through all points that have $\omega_{0 i}>0.00$; i.e. the absolutely unstable region. The saddle point is at $\omega_{s}=0.6570+0.1409 \mathrm{i}, X_{s}=11.05+4.251 \mathrm{i}$. As explained in Section 3.5, $\omega_{s}$ equals $\omega_{g}$ to within order $\epsilon$, which is the degree of non-parallelism in the flow. For this flow, a global analysis gives $\omega_{g}=0.6631+0.1239 \mathrm{i}$. The local analysis is seen to over-predict the growth rate of the linear global mode, which is a common feature of local analyses of wake flows [6,28]. In order to investigate the influence of this discrepancy on the direct and adjoint global modes, we calculate the $k^{+}$and $k^{-}$branches at both values of $\omega_{g}$. These branches are shown in Fig. 2(d,e) for forcing at $\omega_{g}(\mathrm{loc})$ and $\omega_{g}$ (glob). They can be compared with the local wavenumbers, $k$ and $k^{\dagger}$, extracted from the direct and adjoint global modes from the global analysis. These were extracted from the $v_{1}$-eigenfunction at $z=0.79$, where the global mode has the highest absolute value. There are four important points.

Firstly, we confirm that the wavenumber of the direct global mode, $k$ (solid black line), follows $k^{-}$upstream of $X_{c}$ and $k^{+}$ downstream, as already known, and that the wavenumber of the adjoint global mode, $k^{\dagger}$ (dashed black line), follows $k^{+}$upstream of $X_{c}$ and $k^{-}$downstream, as predicted in Section 3.6.

Secondly, the match is closest where the flow is more parallel. For example, when the flow is forced at $\omega_{g}$ (glob), $k^{+}$and $k^{-}$follow $k$ and $k^{\dagger}$ very closely for $X>22.4$, where the flow is nearly parallel, but follow $k$ and $k^{\dagger}$ less closely for $X<22.4$, where the flow is less parallel. Also, the local analysis predicts that the crossing point of the $k^{+}$and $k^{-}$branches is slightly further upstream than that given by the global analysis. This is the case whether or not the flow is forced at $\omega_{g}(\mathrm{loc})$ or $\omega_{g}$ (glob) so is due to the flow's non-parallelism, or to the effect described next, and not due to the discrepancy in $\omega_{g}$.

Thirdly, $k^{-}$and $k^{+}$diverge from $k$ and $k^{\dagger}$ around the upstream boundary. This is not a defect in the local analysis. It is because the global analysis has a Dirichlet boundary condition at $X=0$, while the local analysis assumes that the flow is homogeneous to $X=-\infty$.

Fourthly, the position of maximum structural sensitivity, where $k_{i}^{+}-k_{i}^{-}=0$, is at $X=10.50$ when using $\omega_{g}(\mathrm{loc})$ and $X=10.30$ when using $\omega_{g}$ (glob). These values differ from 11.05, which is the streamwise position of the saddle point in the complex $X$-plane. This small difference is to be expected, for the reasons given in Section 3.7.

The direct global modes obtained from the local analysis at $\omega_{g}(\mathrm{loc})$ are compared with those obtained from the global analysis 
a
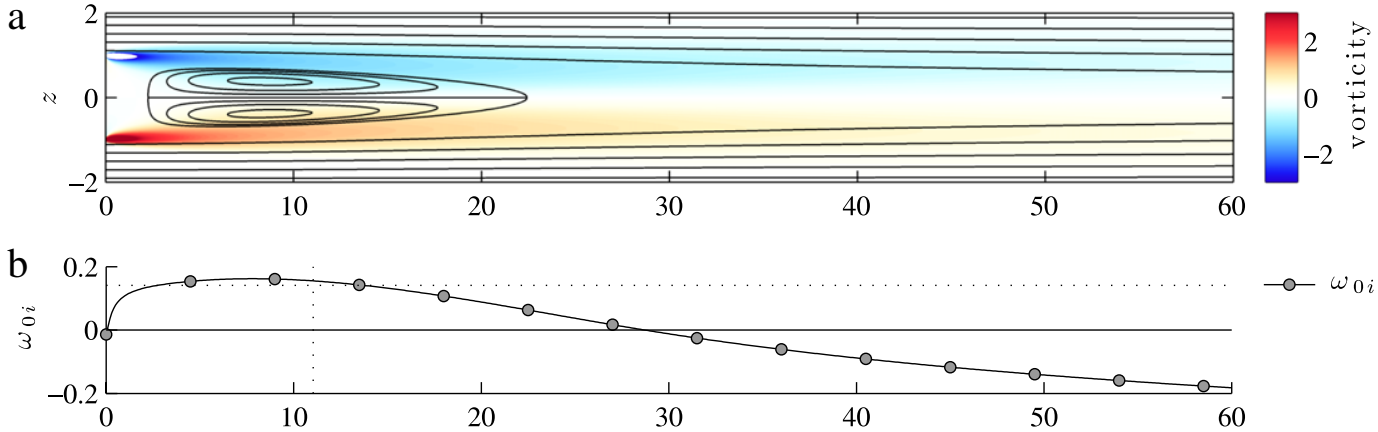

C
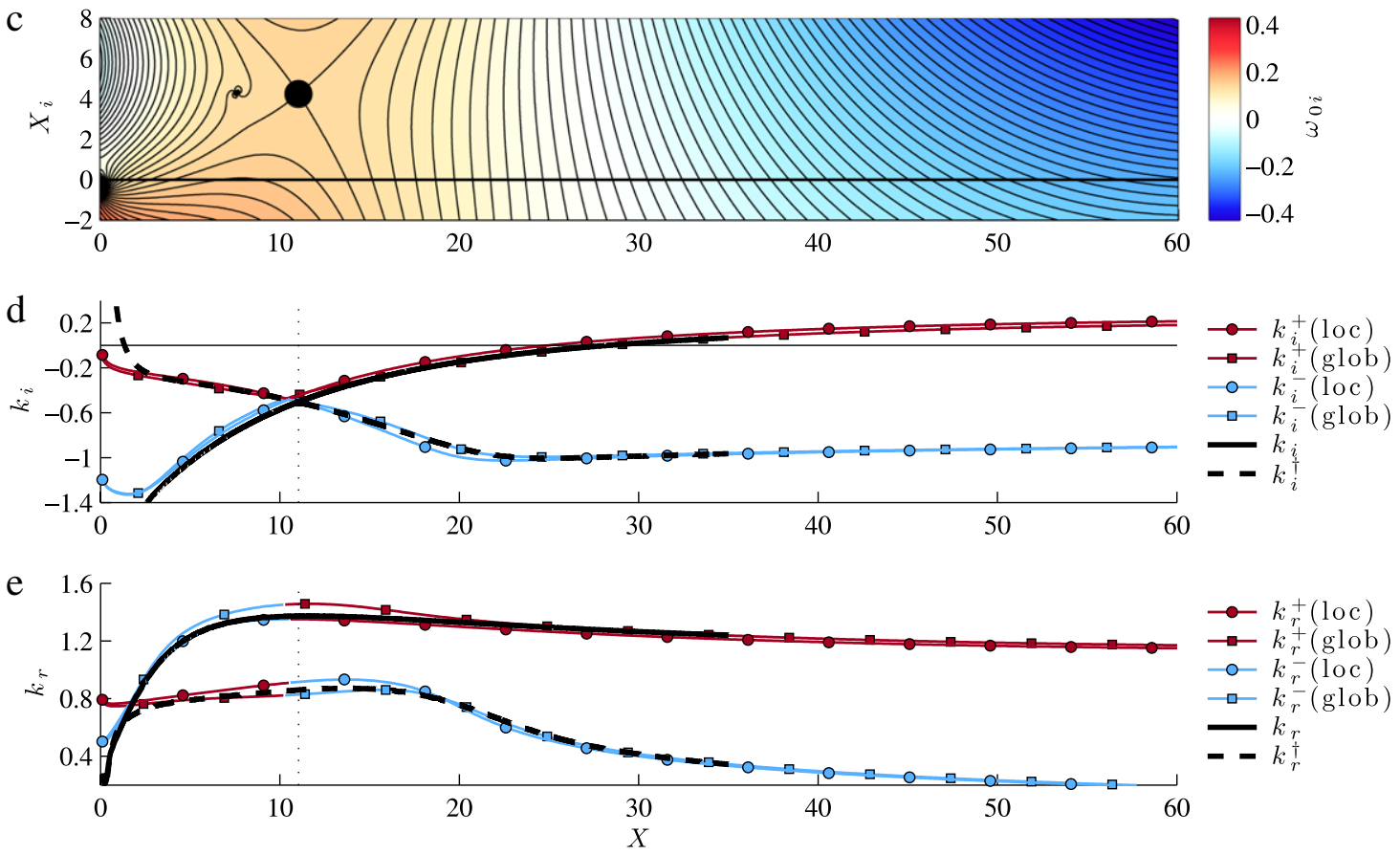

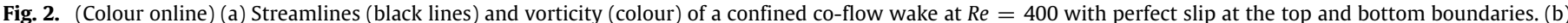

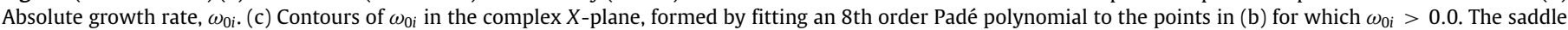

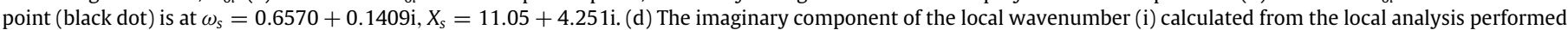

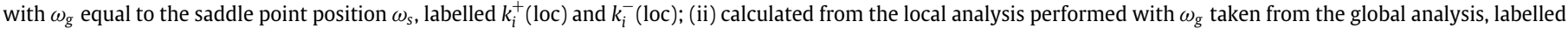

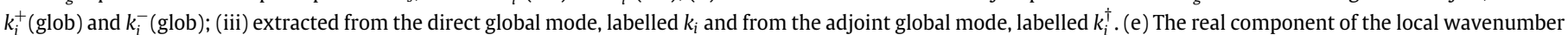
with the same nomenclature as (d).

in Fig. 3(a)-(c). Their structure is identical but the local analysis predicts that the maximum amplitude is reached slightly further upstream than it is in the global analysis. This is due to the overprediction of the growth rate, $\omega_{g}$, as noted by [28]. This can be seen in Fig. 2(d) by the fact that the $k^{+}$branch from the local analysis at $\omega_{g}(\mathrm{loc})$ crosses the $k_{i}$ axis before the $k$ branch from the global analysis, while that at $\omega_{g}$ (glob) crosses at the same place.

The adjoint global modes obtained from the local analysis are compared with those obtained from the global analysis in Fig. 3(d)-(f). They have a similar structure to each other but there are some clear differences around $X=0$, which are due to the different boundary condition there.

The structural sensitivity, as defined by [6] is shown in $3(\mathrm{~g})$. From the global analysis, the maximum of the structural sensitivity is at the position where $\operatorname{Im}\left(k-k^{\dagger}\right)=0$, which is at $X=11.00$. From the local analysis, the maximum of the structural sensitivity is at the position where $\operatorname{Im}\left(k^{+}-k^{-}\right)=0$, which is at $X=$ 10.50. The local analysis predicts the maximum of the structural sensitivity to be slightly further upstream than is predicted by the global analysis. This is the case for both $\omega_{g}(\mathrm{loc})$ and $\omega_{g}$ (glob) and is therefore due to the non-parallelism of the flow or the effect of the upstream boundary condition, and not due to the discrepancy between $\omega_{g}$ (loc) and $\omega_{g}$ (glob). Apart from this small difference, the structural sensitivities are almost indistinguishable. This shows that, for this slowly-developing flow, the structural sensitivity can be estimated easily and accurately with a local stability analysis. If the direct global mode has been calculated with the local analysis, then, apart from a quick calculation to find the eigenfunction in the cross-stream direction, the adjoint has already been calculated.

\subsection{The flow behind a cylinder at $\mathrm{Re}=50$}

Hill [5] and Giannetti and Luchini [6] calculated the direct and adjoint global modes of the two-dimensional flow around a circular cylinder at $R e=50$, based on the cylinder diameter. This is another good test case for the local analysis because there are several published results and it is less parallel than the previous test case.

Fig. 4(a) shows the streamlines and vorticity of this flow and Fig. 4(b) shows the absolute growth rate $\omega_{0 i}$ as a function of downstream distance. Fig. 4(c) shows the position of the saddle point $\omega_{s}$ in the complex $X$-plane, which was calculated by fitting Padé polynomials through all points downstream of the cylinder with $\omega_{0 i}>$ 0.03 . The saddle point is at $\omega_{s}=0.791+0.083 \mathrm{i}, X_{s}=1.297+$ 

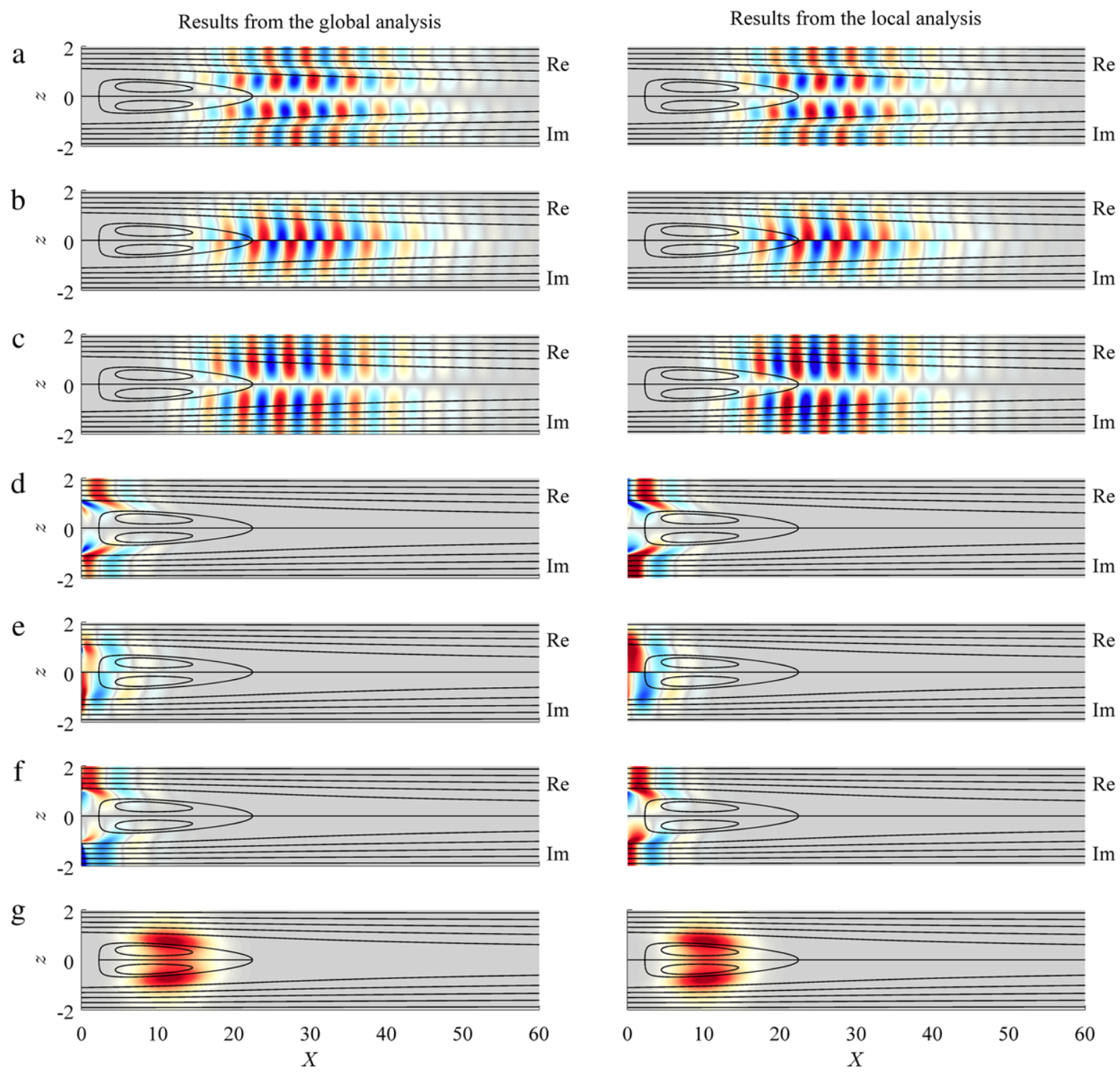

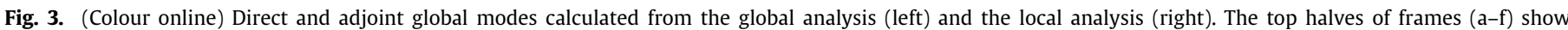

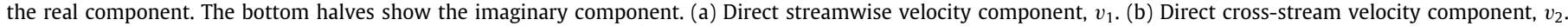

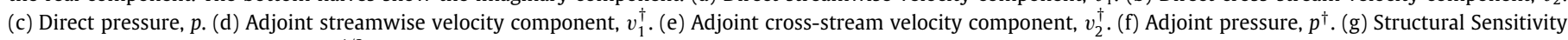
$\nabla_{L} \omega_{m}=\left(\left(v_{1}^{2}+v_{2}^{2}\right) \times\left(v_{1}^{\dagger 2}+v_{2}^{\dagger 2}\right)\right)^{1 / 2}$.

0.699i. The threshold of 0.03 was chosen because a threshold of 0.00 gave rise to too many nearby saddle points. For comparison, the global analysis of [6] gives $\omega_{g}=0.750+0.013 \mathrm{i}$ and the local analysis of [30] gives $\omega_{g}=0.785+0.091 \mathrm{i}$. The $k^{+}$and $k^{-}$ branches are shown in Fig. $4(\mathrm{~d})-(\mathrm{e})$ using $\omega_{g}$ (local) and $\omega_{g}$ (global).

Fig. 5(a)-(b) shows the vorticity of the direct global mode obtained from the local analysis and can be compared directly with Fig. 2 of [5], which is obtained from the global analysis. Fig. 5(c)-(d) shows the vorticity of the adjoint global mode obtained from the local analysis and can be compared directly with Fig. 3 of [5]. The local results are close to the global results in the region behind the cylinder but differ in the region around the cylinder. This is not surprising because the flow is strongly non-parallel there.

The structural sensitivity is shown in Fig. 6, and can be compared directly with Fig. 17 of [6]. Both frames are calculated from the local analysis but the left frame is calculated at $\omega_{g}$ (local), while the right frame is calculated at $\omega_{g}$ (global). The local analysis predicts the same features as the global analysis but there are some noticeable differences. Firstly, the centre of the structural sensitivity is too far upstream when $\omega_{g}($ local $)$ is used. This was also seen for the wake flow in Section 5.1 and is because the local analysis overpredicts the growth rate. Secondly, the $z$-dependence of the global mode is poorly predicted in regions where the flow is strongly nonparallel, such as at the end of the recirculation zone. Nevertheless, this shows that the local analysis can estimate the structural sensitivity in this type of flow, at very little computational cost.

It is worth mentioning that we also attempted to use this method to compute the global modes for the swirling vortex breakdown bubble in [31], which is very non-parallel around the vortex breakdown bubble. It was impossible to identify the $k^{+}$and $k^{-}$branches in the non-parallel region and therefore impossible to generate direct and adjoint global modes.

\section{Conclusions}

In an unstable open flow, it is useful to know which regions are most receptive to forcing and which regions are most sensitive to changes in internal feedback. These regions can be found easily if the direct and adjoint global modes have been calculated. 
a

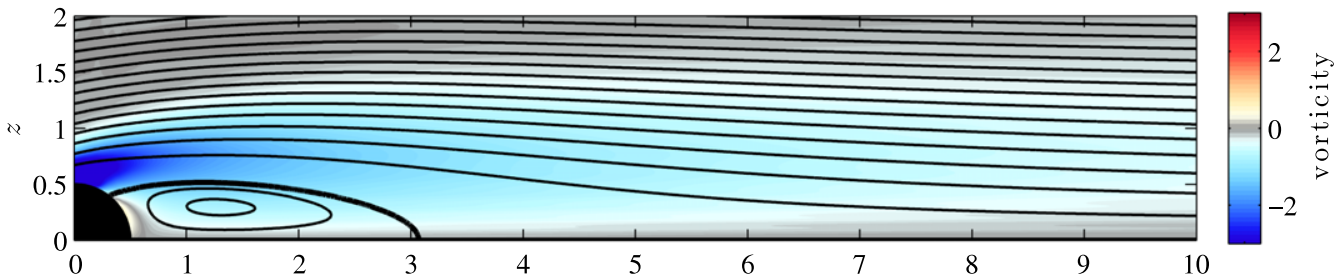

b
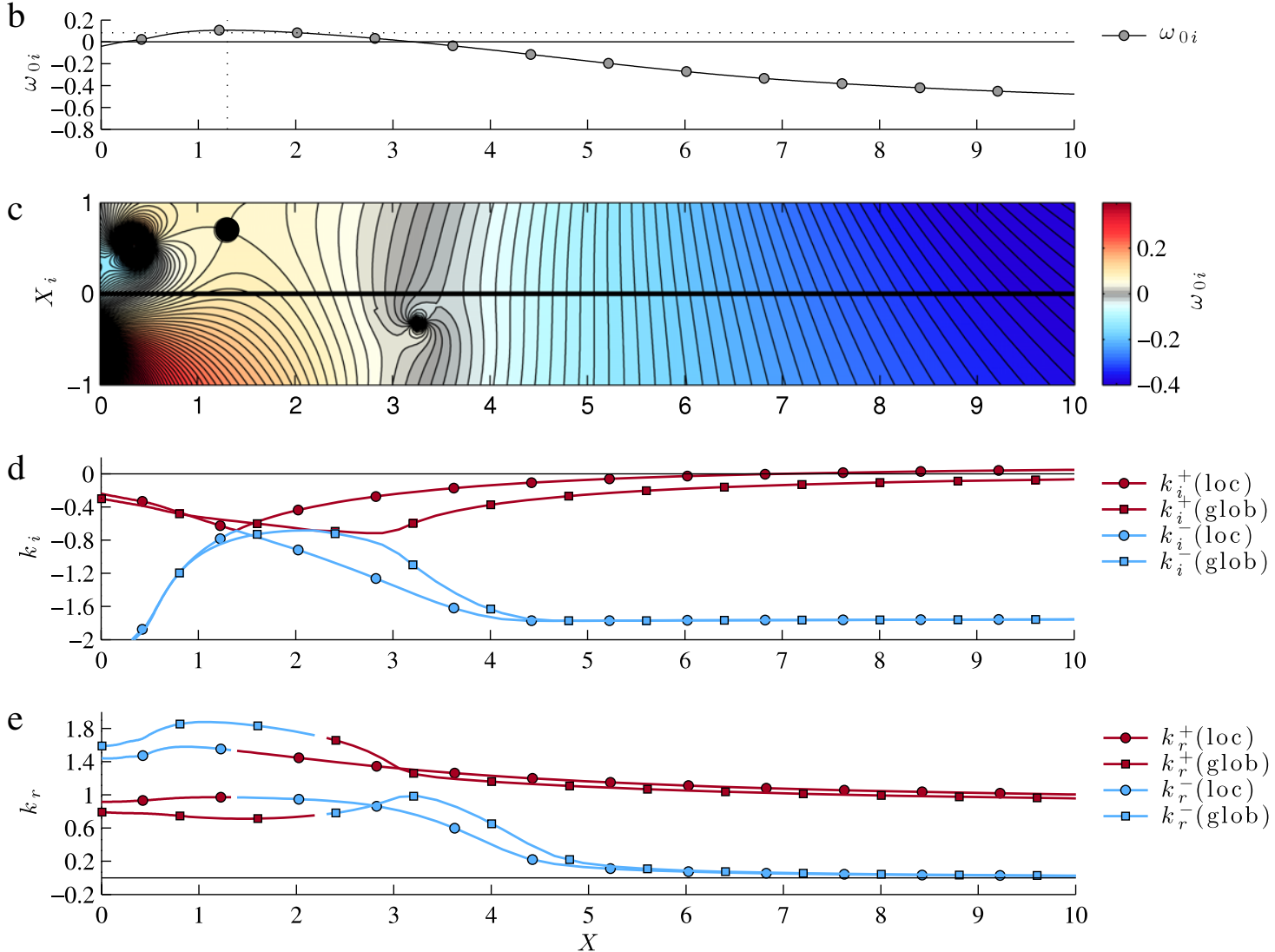

Fig. 4. As for Fig. 2 but for the flow around a cylinder at $R e=50$.
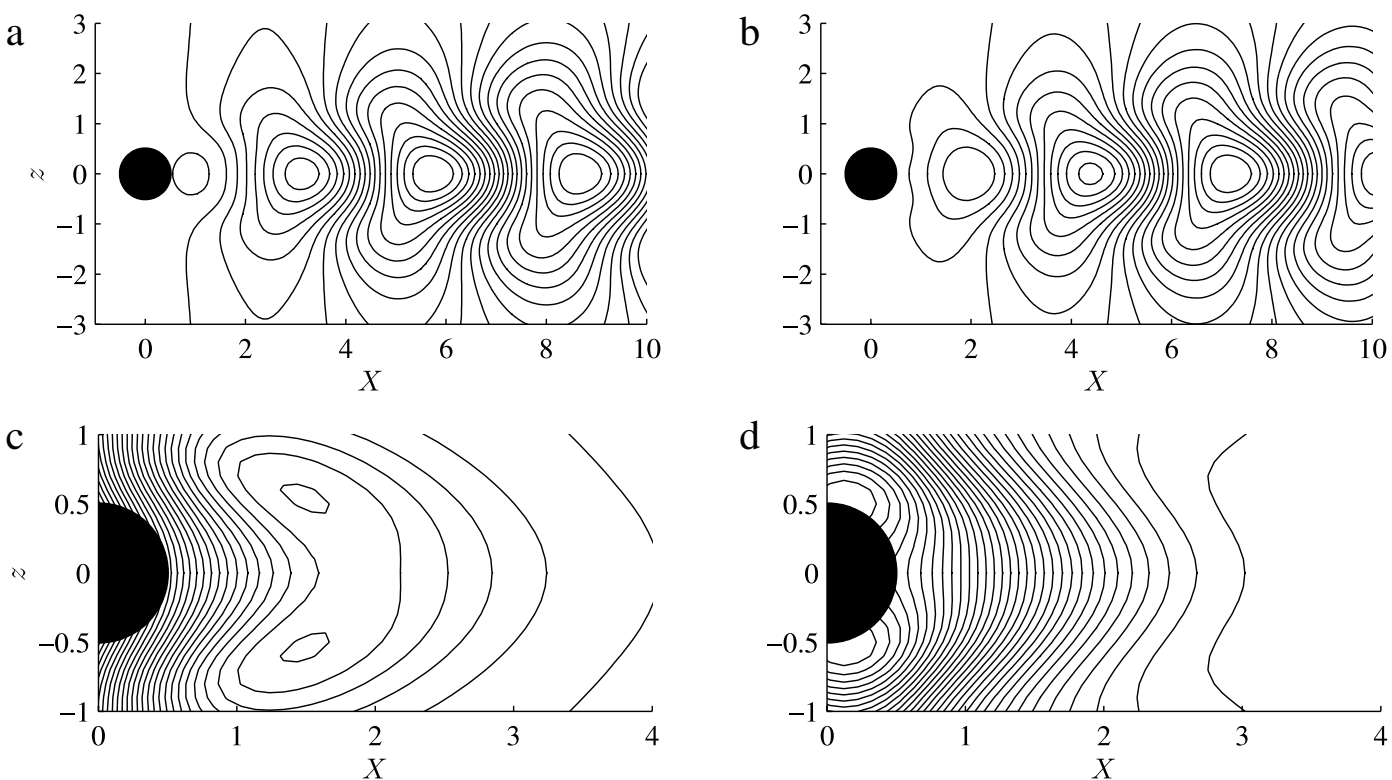

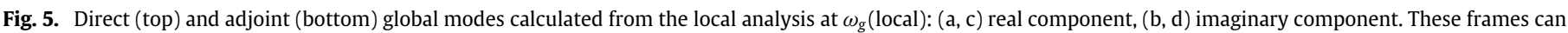
be compared with Figs. 2 and 3 of [5]. 

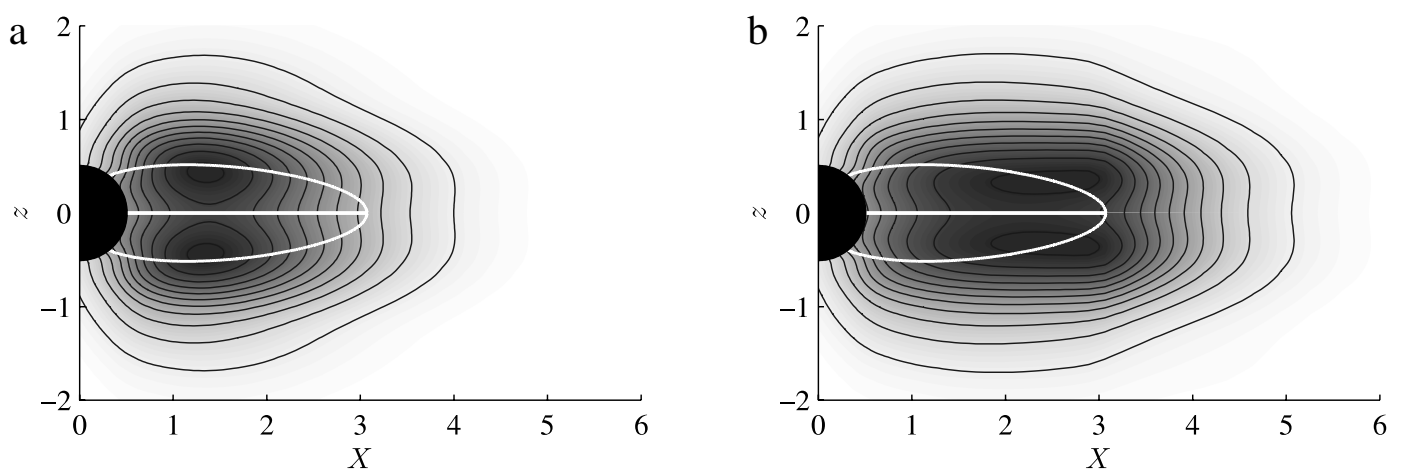

Fig. 6. The structural sensitivity calculated from the local analysis at (a) $\omega_{g}$ (local) and (b) $\omega_{g}$ (global). These frames can be compared with Fig. 17 of [6].

These modes are usually calculated with a global linear stability analysis, meaning that small perturbations on top of a base flow are discretized on a 2D or 3D grid. This creates a generalized matrix eigenvalue problem, which is then solved numerically. These matrices can have millions of degrees of freedom, so this procedure is computationally expensive and is impractical for many flows.

An alternative approach, which is applicable to weakly nonparallel open flows, is to calculate the direct global mode with a local stability analysis. This is orders of magnitude cheaper than a global analysis. The main result of this paper is to show that the adjoint global mode then follows at almost no extra cost. We show this formally for the Ginzburg-Landau equation and find that the direct global mode is formed from the $k^{-}$-branch upstream and the $k^{+}$-branch downstream, while the adjoint global mode is formed from the $k^{+}$-branch upstream and the $k^{-}$-branch downstream. We include higher order terms of the WKBJ analysis in order to show that these analytical relationships are valid up to order $\epsilon$, which measures the non-parallelism of the flow. Furthermore, we show that the maximum of the structural sensitivity, as defined by [6], is the point at which the spatial branches, $k^{+}$and $k^{-}$, have identical imaginary components.

We apply this to the linearized Navier-Stokes (LNS) equations and show that, if the direct global mode has already been calculated, the only extra cost in calculating the adjoint mode is in calculating the adjoint eigenfunction at each point in the flow. This cost is small because the adjoint eigenvalue is already known.

We compare the local and global results for two flows: a confined wake flow at $R e=400$, and the flow behind a cylinder at $R e=50$. The procedure works very well for the confined wake flow: the local wavenumbers of the direct and adjoint global modes closely follow the $k^{+}$and $k^{-}$branches of the local analysis, as expected, and the structural sensitivity calculated with the local analysis is almost indistinguishable from that calculated with the global analysis. The procedure works less well for the cylinder: although the local and global results are qualitatively similar, the local analysis over-predicts the growth rate and therefore predicts that the wavemaker region lies too far upstream. We conclude that the procedure works less well for the cylinder because the wavemaker sits in a region that is less parallel.

Some flows, such as those in a gas turbine fuel injector [23], contain more than one unstable global mode. Each of these global modes has, in a local analysis, an associated saddle point of $\omega(k)$ in the $k$-plane and an associated saddle point of $\omega_{0}(X)$ in the $X$ plane. Therefore the technique in this paper can be applied to each of these saddle points individually and can identify multiple global modes in a flow, if they exist.

In many real flows, the assumptions underlying the WKBJ approach are not met very closely. Nevertheless, the techniques of local stability analysis have proved to be remarkably robust, probably because the wavemaker region often lies in a region of nearly parallel flow. In such cases, a local stability analysis will give reasonable estimates of the direct and adjoint global modes at much less computational cost than a global analysis. For example, the global modes of a 3D flow that evolves slowly in the streamwise direction could be calculated by combining the results of several 2D calculations. This opens the door to the application of sensitivity analysis to flows that are currently beyond the reach of global analysis.

\section{Acknowledgements}

We gratefully acknowledge the help of Outi Tammisola, who provided the confined wake flow and the corresponding global stability analysis. This work was supported by the European Research Council under Project ALORS 2590620 and by MPJ's visiting Professorship at École centrale de Lyon.

\section{References}

[1] P.A. Monkewitz, The absolute and convective nature of instability in twodimensional wakes at low Reynolds numbers, Phys. Fluids 31 (1998) 999-1006.

[2] J.-M. Chomaz, Global instabilities in spatially developing flows: non-normality and nonlinearity, Annu. Rev. Fluid Mech. 37 (2005) 357-392.

[3] J.-M. Chomaz, Linear and nonlinear, local and global stability analysis of open flows, in: Turbulence in Spatially Extended Systems, Nova, ISBN: 1-56072120-0, 1993.

[4] D. Sipp, O. Marquet, P. Meliga, A. Barbagallo, Dynamics and control of global instabilities in open flows: a linearized approach, Appl. Mech. Rev. 63 (2010) 030801.

[5] D.C. Hill, A theoretical approach for analyzing the restabilization of wakes, AIAA Paper 92-0067, and NASA Tech. Mem. 103858, 1992.

[6] F. Giannetti, P. Luchini, Structural sensitivity of the first instability of the cylinder wake, J. Fluid Mech. 581 (2007) 167-197.

[7] F. Giannetti, P. Luchini, Receptivity of the circular cylinders first instability, in: 5th Eur. Fluid Mech. Conf., Toulouse, 2003.

[8] P. Luchini, F. Giannetti, J.O. Pralits, Structural sensitivity of linear and nonlinear global modes, AIAA Paper 2008-4227, 2008.

[9] O. Marquet, D. Sipp, L. Jacquin, J.-M. Chomaz, Multiple Time Scale Analysis and Sensitivity Analysis for the Passive Control of the Cylinder Flow, AIAA, 2008, pp. 2008-4228.

[10] O. Marquet, D. Sipp, L. Jacquin, Sensitivity analysis and passive control of cylinder flow, J. Fluid Mech. 615 (2008) 221-252.

[11] O. Marquet, M. Lombardi, J-M. Chomaz, D. Sipp, L. Jacquin, Direct and adjoint global modes of a recirculation bubble: lift-up and convective nonnormalities, J. Fluid Mech. 622 (2009) 1-21.

[12] P. Meliga, J-M. Chomaz, D. Sipp, Unsteadiness in the wake of disks and spheres: Instability, receptivity and control using direct and adjoint global stability analyses, J. Fluids Struct. 25 (2009) 601-616.

[13] P. Meliga, D. Sipp, J-M. Chomaz, Open-loop control of compressible afterbody flows using adjoint methods, Phys. Fluids 22 (2010) 054109.

[14] A. Barbagallo, G. Dergham, D. Sipp, P. Schmid, J.-C. Robinet, Closed-loop control of unsteadiness over a rounded backward-facing step, J. Fluid Mech. 703 (2012) 326-362.

[15] L. Marino, P. Luchini, Adjoint analysis of the flow over a forward-facing step, Theor. Comput. Fluid Dyn. 23 (2009) 37-54.

[16] O. Tammisola, F. Lundell, P. Schlatter, A. Wehrfritz, L.D. Söderberg, Global linear and nonlinear stability of viscous confined plane wakes with co-flow, J. Fluid Mech. 675 (2011) 397-434. 
[17] O. Tammisola, Oscillatory sensitivity patterns for global modes in wakes, J. Fluid Mech. 701 (2012) 251-277.

[18] P. Meliga, F. Gallaire, J-M. Chomaz, A weakly nonlinear mechanism for mode selection in swirling jets, J. Fluid Mech. (2012) http://dx.doi.org/10.1017/jfm. 2012.93.

[19] P. Huerre, P.A. Monkewitz, Local and global instabilities in spatially developing flows, Annu. Rev. Fluid Mech. 22 (1990) 473-537.

[20] P.A. Monkewitz, P. Huerre, J-M. Chomaz, Global linear stability analysis of weakly non-parallel shear flows, J. Fluid Mech. 251 (1993) 1-20.

[21] B. Pier, Local and global instabilities in the wake of a sphere, J. Fluid Mech. 603 (2008) 39-61.

[22] K. Oberleithner, M. Sieber, C.N. Nayeri, C.O. Paschereit, C. Petz, H.-C. Hege, B.R. Noack, Three-dimensional coherent structures in a swirling jet undergoing vortex breakdown: stability analysis and empirical mode construction, J. Fluid Mech. 679 (2011) 383-414.

[23] M.P. Juniper, Absolute and convective instability in gas turbine fuel injectors, ASME Turbo Expo. June 2012, Copenhagen, Denmark, GT2012-68253, 2012.
[24] D.C. Hill, Adjoint systems and their role in the receptivity problem for boundary layers, J. Fluid Mech. 292 (1995) 183-284.

[25] C.M. Bender, S.A. Orszag, Advanced Mathematical Methods for Scientists and Engineers: Asymptotic Methods and Perturbation Theory, Springer, 1978.

[26] B. Pier, Signalling problem in absolutely unstable systems, Theor. Comput. Fluid Dyn. 25 (2011) 7-17.

[27] L.D. Landau, E.M. Lifshitz, Physical Kinetics, Butterworth-Heinenann, 1981.

[28] M.P. Juniper, O.L. Tammisola, F. Lundell, The local and global stability of confined planar wakes at intermediate Reynolds number, J. Fluid Mech. 686 (2011) 218-238.

[29] T. Herbert, Parabolized stability equations, Annu. Rev. Fluid Mech. 29 (1997) $245-283$.

[30] B. Pier, On the frequency selection of finite-amplitude vortex shedding in the cylinder wake, J. Fluid Mech. 458 (2002) 407-417.

[31] U. Qadri, D. Mistry, M.P. Juniper, Structural sensitivity of spiral vortex breakdown, J. Fluid Mech. 720 (2013) 558-581. 